\title{
Collisionless shock formation and X-ray emission around supernova shock breakout
}

\author{
Gwenael Giacinti* \\ University of Oxford, Clarendon Laboratory, Parks Road, Oxford OXI 3PU, United Kingdom \\ E-mail: gwenael.giacintiephysics.ox.ac.ulk
}

\section{Anthony R. Bell}

University of Oxford, Clarendon Laboratory, Parks Road, Oxford OXI 3PU, United Kingdom

E-mail: t.bell1ephysics.ox.ac.ult

\begin{abstract}
We study analytically and numerically the formation of the collisionless shock around supernova shock breakout. Following core collapse, a radiation-dominated shock travels through the supernova progenitor. The collisionless shock (CS) is usually expected to form during breakout, when the radiation-dominated shock reaches the optically thin layers of the progenitor. In this work, we show that for some progenitors surrounded with optically thick winds, the collisionless shock forms before breakout. An X-ray flash would occur at shock breakout, even for 'slow' shocks. High-energy neutrinos with $E \gtrsim 100 \mathrm{GeV}-1 \mathrm{TeV}$ would precede the photon flash by typically a few minutes (Wolf-Rayet progenitors), up to $\sim 10$ hours (red supergiants) [四]. SN 2008D/XRF 080109 may have been an event for which a CS is formed before breakout.
\end{abstract}

Swift: 10 Years of Discovery

2-5 December 2014

La Sapienza University, Rome, Italy

\footnotetext{
* Speaker.
} 


\section{Introduction}

During a core-collapse supernova (SN), a radiation-dominated shock (RDS) propagates inside the (optically thick) hydrostatic core of the progenitor. The pressure in the downstream of the shock is dominated by radiation pressure [ [, , []]. The width of a RDS $\left(\simeq \lambda c / 3 u_{\mathrm{s}}\right.$, where $u_{\mathrm{s}}$ is the shock velocity and $\lambda$ the photon mean free path) is larger than the gyroradius of suprathermal particles, which therefore cannot be accelerated via diffusive shock acceleration. When the shock reaches the optically thin outer layers of the star, photons cannot remain confined in its immediate downstream, and escape, emitting a flash of photons -shock breakout (SB). The RDS then stalls.

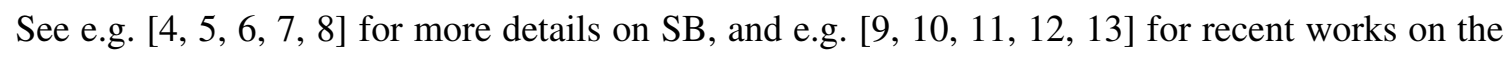

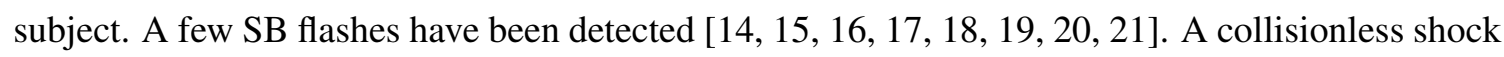
(CS) is expected to form later [ [5, Q6, [22, [23]. Once the CS is formed, particle acceleration may start. Some SN progenitors are thought to shed mass at a remarkable rate prior to the explosion [24], and some are likely to be surrounded with optically thick winds [25, 26]. For the case of an optically thick wind, shock breakout occurs in the wind, at an optical depth $\tau_{\mathrm{br}}$ approximately equal to $c / u_{\mathrm{S}}=\beta_{\mathrm{s}}^{-1}[\mathrm{G}]$. A CS must appear during SB [27, 28, 20, [3], [1], [2]].

In this study, we demonstrate that the CS forms before shock breakout for some progenitors enshrouded in thick winds [U]. We predict that for such progenitors, X-rays would be emitted from the very beginning of shock breakout, even for 'slow' shocks. We find that supernova SN 2008D/XRF 080109, discovered by Swift [ए్]], may be an explosion where the CS formed before shock breakout -see Section 4 .

\section{Formation of a collisionless shock before supernova shock breakout from a thick wind}

For progenitors surrounded with optically thin winds, the flash of photons at SB accelerates the low density circumstellar medium to a velocity about $\propto r^{-2}$ ( $r$ is the distance to the center of the progenitor). The shocked outer layers of the star then push supersonically into the more slowly moving circumstellar material at larger $r$, which leads to the formation of a collisionless shock. We verified, with the 1D spherical radiation-hydrodynamics code presented in [四], that a CS forms after SB for a progenitor in an optically thin wind. In this case, the RDS stalls when entering optically thin material.

However, in some situations, the RDS can also stall inside optically thick material, and lead to the formation of a (radiative) CS. We find that this happens for some supernovae exploding in optically thick winds. For thick winds, shock breakout occurs at a radius $r_{\mathrm{br}}$, which is larger than the radius of the star $r_{*}$. When the progenitor is surrounded with a very dense wind, such as for Type IIn SNe, the RDS survives the transition from the core to the thick wind at $r=r_{*}$. On the contrary, we find that when the wind is only moderately thick, the RDS stalls when exiting the hydrostatic core at $r=r_{*}$, and a radiative CS forms in the wind at $r_{*}<r<r_{\mathrm{br}}$, before SB (i.e. before photons start to escape from the thick layers of the wind).

Let us consider two concentric shells in the thick wind, with respective radii $r_{1}(t)$ and $r_{2}(t)$,

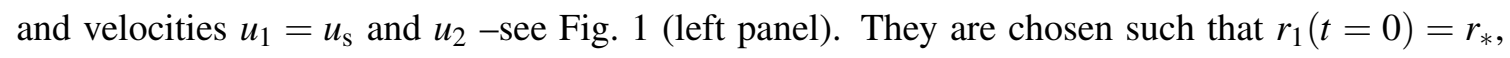
and $r_{*}<r_{2}(t=0)<r_{*}+\lambda / \beta_{\mathrm{s}}$, where $\lambda$ is the photon mean free path in the wind at $r \simeq r_{*}$. In 

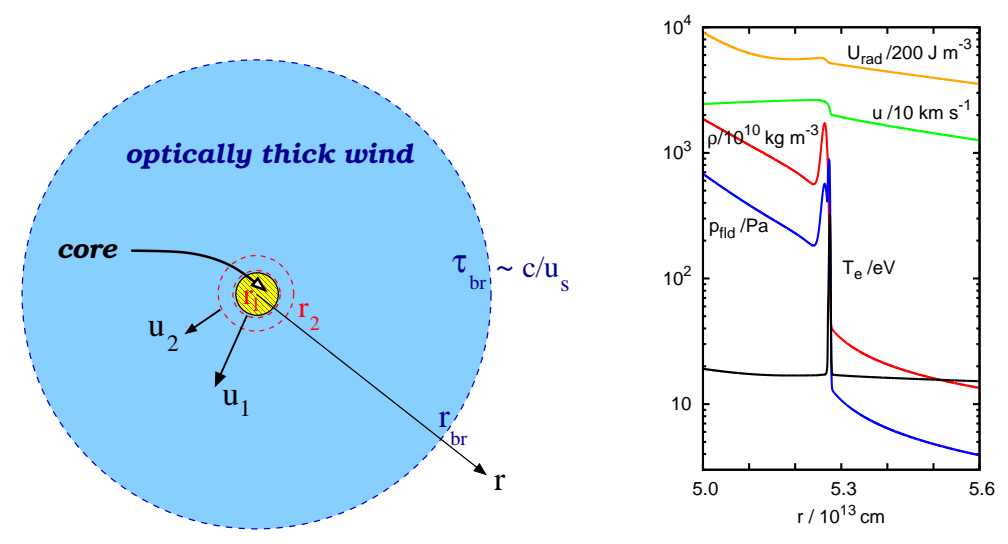

Figure 1: Left panel: Schematic description of the problem. A star with radius $r_{*}=r_{1}(t=0)$ (yellow) is surrounded with an optically thick wind (part at $r<r_{\mathrm{br}}$ in blue). Shock breakout occurs at $r_{\mathrm{br}} \gg r_{*}$. The density of the core is $\gg$ than the density of the surrounding wind. The radiation-dominated shock leaves the core and enters the wind at $t=0$. A collisionless shock forms when the shell at $r_{1}(t)$ hits supersonically the shell at $r_{2}(t)\left(r_{2}(0)>r_{1}(0)\right)$, which can happen at $r<r_{\mathrm{br}}$, before breakout; Right panel: Simulation of a red supergiant exploding in a thick wind. Zoom around the region where the CS appears (discontinuity in the green -velocity- curve around $r \approx 5.3 \cdot 10^{13} \mathrm{~cm}$, at $\approx r_{\mathrm{br}} / 2$ and $\approx 1.6 r_{*}$ for this simulation). Spikes in fluid pressure $p_{\text {fld }}$ and electron temperature $T_{\mathrm{e}}$ can be seen in the CS immediate downstream.

the limiting case where all photons that have accelerated the shell at $r_{1}$ also pass through the shell at $r_{2}$ (no absorption), the velocity reached by the latter shell cannot exceed $u_{2} \leq u_{1}\left(r_{*} / r_{2}(0)\right)^{2}+$ $\kappa E_{\mathrm{r}} / 4 \pi c r_{2}^{2}(0)$, where $\kappa$ is the opacity and $E_{\mathrm{r}} \simeq \int_{r_{*}}^{r_{2}(0)} 4 \pi r^{2} \frac{1}{2 \kappa \lambda} u_{\mathrm{S}}^{2} d r$ is an upper limit on the energy that can be radiated through the shell at $r_{2}$ by the fluid between $r_{1}$ and $r_{2}$. If $E_{\mathrm{r}}$ is sufficiently large, it can compensate for the $\left(r_{*} / r_{2}(0)\right)^{2}$ factor : $u_{2}$ then remains $\geq u_{1}$ and the RDS survives in the wind. If the wind between the two shells cannot radiate enough photons through the shell at $r_{2}$ to compensate for the dilution of photons due to shock curvature, then the shell at $r_{1}$ can catch up and hit supersonically the shell at $r_{2}$, at a radius $r<r_{\text {br }}$ (i.e. before SB). A CS then forms before shock breakout. One can show that this happens when [四]: $\beta_{\mathrm{s}} \lesssim 10 \lambda / r_{*}$. For a $r^{-2}$ wind, this condition becomes :

$$
\beta_{\mathrm{s}} \lesssim 0.1\left(\frac{u_{\mathrm{w}}}{10 \mathrm{~km} / \mathrm{s}}\right)\left(\frac{r_{*}}{10^{13} \mathrm{~cm}}\right)\left(\frac{5 \cdot 10^{-4} \mathrm{M}_{\odot} / \mathrm{yr}}{\dot{M}}\right)
$$

where $u_{\mathrm{w}}$ and $\dot{M}$ are respectively the wind velocity and the mass-loss rate of the progenitor. WolfRayet stars and red supergiants with relatively large $\dot{M}$ before the explosion are good candidates, but not progenitors of Type IIn SNe. SN 2008D may satisfy this inequality, see discussion in Section 4. For progenitors with wind densities $\propto r^{-2}$, this corresponds $r_{\mathrm{br}} \approx 10 r_{*}$. However, this scenario can also be satisfied for progenitors with $r_{\mathrm{br}} / r_{*} \gg 10$, if, for example, the wind density profile is flatter than $r^{-2}$ at $r<r_{\mathrm{br}}$, due to a change in the mass-loss rate with time.

We verified this with our radiation-hydrodynamics code : Fig. (right panel) shows the formation of a CS before breakout for a red supergiant exploding in a thick wind, see caption for details. Once formed, the CS continues to propagate to $r \gg r_{\mathrm{br}}$. 


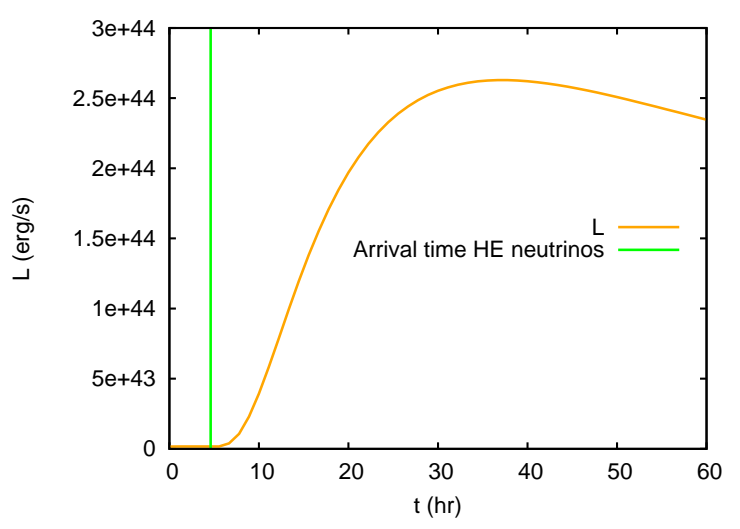

Figure 2: Simulation of a red supergiant exploding in a thick wind, see text for parameters. Orange line for the luminosity $\mathrm{L}(\mathrm{t})$. The green line shows the arrival time of the first secondary $\sim \mathrm{TeV}$ neutrinos from particles accelerated at $r<r_{\mathrm{br}}$. Around the peak, and at later times, most of the energy is radiated in X-rays, which are emitted by the CS propagating, at these times, in the thin part of the wind at $r>r_{\mathrm{br}}$.

\section{Particle acceleration and high-energy neutrinos preceding an X-ray flash}

When the CS forms before shock breakout, it injects X-ray photons with energies $\gtrsim(1-$ 10) $\mathrm{keV}$ in the optically thick wind. Thus, we predict that for progenitors following our scenario, the photon flash at breakout will contain X-rays since the very beginning of SB, even for 'slow' shocks. These hard photons reflect the presence of the hot downstream of the CS. If no CS were present before SB, the first photons to escape at SB would be softer : $\sim(10-100) \mathrm{eV}$ for a 'slow' $\operatorname{RDS}\left(\beta_{\mathrm{s}} \approx 0.1\right)$.

We have shown in [四] that particles can be accelerated at the CS, at $r<r_{\mathrm{br}}$, via diffusive shock acceleration. Energies $E \gtrsim(1-10) \mathrm{TeV}$ can be reached. Secondary high-energy neutrinos are then produced in the optically thick layers of the wind and can reach the observer before the flash of photons at breakout. For an event following this scenario and occurring in the Milky Way or in the Magellanic Clouds, high-energy neutrinos would be detectable by IceCube before photons from breakout [四]. More neutrinos would be produced later, in the post-shock breakout phase.

We simulate the explosion of a red supergiant with $r_{*}=10^{13} \mathrm{~cm}$ and $\beta_{\mathrm{s}} \simeq 0.09$, in a thick wind with density $\propto r^{-2}$ and $r_{\mathrm{br}} \simeq 10^{14} \mathrm{~cm}$. We show in Figure $\rrbracket$ the evolution with time of its luminosity $\mathrm{L}$. The arrival time of the first $\sim \mathrm{TeV}$ neutrinos, for a given observer, is shown in green : In this case, secondary neutrinos arrive several hours before SB. For a Wolf-Rayet progenitor, they would rather arrive a few minutes before SB.

\section{SN 2008D / XRF 080109}

The X-ray flash XRF 080109, observed by Swift [ए耳], has been suggested to be the signature of shock breakout for Type Ibc supernova SN 2008D. We suggest here that the CS may have formed before SB for SN 2008D/XRF 080109. Svirski \& Nakar [34] proposed that the progenitor was a Wolf-Rayet star surrounded with a moderately thick wind $\left(r_{\mathrm{br}} \lesssim 10 r_{*}\right)$. They suggested that the progenitor underwent enhanced mass-loss prior to the explosion, for $\lesssim 10 \mathrm{~d}$, with mass-loss rate 
$\dot{M} \approx 2 \cdot 10^{-4} \mathrm{M}_{\odot} \mathrm{yr}^{-1}$ and wind velocity $u_{\mathrm{w}} \approx 1000 \mathrm{~km} \mathrm{~s}^{-1}$. For a shock velocity $\beta_{\mathrm{s}} \approx 0.25$, and progenitor radius $r_{*} \approx 10^{11} \mathrm{~cm}, \mathrm{SN} 2008 \mathrm{D}$ would satisfy Inequality (‥J), making it a possible candidate for our theory.

This may ease the tension between duration of the flash of photons, and radiated energy (e.g. [[1]]). A relatively 'low' flux of photons at SB would be consistent with our scenario, where a fraction of the energy would already be in the thermal plasma in the downstream of a CS, when the shock starts to reach $r_{\mathrm{br}}$.

A more detailed modelling of the spectrum a breakout should lead to a better understanding of when the radiative CS of SN 2008D was formed.

\section{Conclusions and perspectives}

For a SN occurring in an optically thin wind, the collisionless shock always forms during or after shock breakout, which implies that particle acceleration cannot start before the beginning of the photon flash.

We have shown here that for some stars exploding in optically thick winds, the RDS stalls in the wind at $r<r_{\mathrm{br}}$, and a radiative CS starts to appear before $\mathrm{SB}$, within the remains of the former RDS. See condition in Section 2. We find that particles can be accelerated to $\gtrsim \mathrm{TeV}$ energies at the CS. If such an event were to occur within $\sim 100 \mathrm{kpc}$ from Earth, IceCube would be able to detect secondary high-energy neutrinos (from these cosmic rays), arriving before the flash of photons at shock breakout $: \approx$ a few minutes before SB for Wolf-Rayets, up to $\sim 10$ hours for red supergiants.

A CS may have formed before SB for SN 2008D/XRF 080109, assuming a progenitor with the parameters proposed in Reference [34]. This gives another important reason to search for SN 2008D-like events in the future. One can use them to study the formation times of CS with respect to shock breakout, and understand better the onset of particle acceleration and magnetic field amplification at supernovae occurring in dense winds. Higher energies are reached after SB : Protons (and nuclei) are expected to be accelerated to energies $E \gtrsim \mathrm{PeV}$ during the first few days or decades following a SN in a dense circumstellar wind [33].

\section{Acknowledgments}

This work was funded by the European Research Council under the European Community's Seventh Framework Programme (FP7/2007 - 2013) / ERC Grant agreement No. 247039.

\section{References}

[1] G. Giacinti and A. R. Bell, Collisionless shocks and TeV neutrinos before supernova shock breakout from an optically thick wind, MNRAS 449 (2015) 3693 [arXiv:1503.04170].

[2] Y. B. Zel'dovich and Y. P. Raizer, Physics of shock waves and high-temperature hydrodynamic phenomena, Academic Press, New York 1966.

[3] T. A. Weaver, The structures of supernova shock waves, ApJ Suppl. Ser. 32 (1976) 233.

[4] S. A. Colgate, ApJ 187 (1974) 321. 
[5] R. A. Chevalier and R. I. Klein, Nonequilibrium processes in the evolution of type II supernovae, ApJ 234 (1979) 597.

[6] L. Ensman and A. Burrows, Shock breakout in SN 1987A, ApJ 393 (1992) 742.

[7] C. D. Matzner and C. F. McKee, The expulsion of stellar envelopes in core-collapse supernovae, ApJ 510 (1999) 379 [astro-ph/9807046].

[8] S. Blinnikov, P. Lundqvist, O. Bartunov, K. 'i. Nomoto and K. Iwamoto, Radiation hydrodynamics of sn 1987a: I. global analysis of the light curve for the first 4 months, ApJ 532 (2000) 1132 [astro-ph/9911205].

[9] B. Katz, R. Budnik and E. Waxman, Fast radiation mediated shocks and supernova shock breakouts, ApJ 716 (2010) 781 [arXiv:0902.4708].

[10] E. Nakar and R. 'e. Sari, Early supernovae light-curves following the shock-breakout, ApJ 725 (2010) 904 [arXiv:1004.2496].

[11] B. Katz, N. Sapir and E. Waxman, Non-relativistic radiation mediated shock breakouts: II. Bolometric properties of SN shock breakout, ApJ 747 (2012) 147 [arXiv:1103.5276].

[12] N. Sapir, B. Katz and E. Waxman, Non-relativistic radiation mediated shock breakouts: I. Exact bolometric planar breakout solutions, ApJ 742 (2011) 36 [arXiv:1103.5075].

[13] N. Sapir, B. Katz and E. Waxman, Non-relativistic radiation mediated shock breakouts: III. Spectral properties of SN shock breakout, ApJ 774 (2013) 79 [arXiv:1304.6428].

[14] S. Campana, V. Mangano, A. J. Blustin, P. Brown, D. N. Burrows, G. Chincarini, J. R. Cummings,

G. Cusumano et al., The shock break-out of grb 060218/sn 2006aj, Nature 442 (2006) 1008 [astro-ph/0603279].

[15] S. Gezari, L. Dessart, S. Basa, D. C. Martin, J. D. Neill, S. E. Woosley, D. J. Hillier, P. Astier et al., Probing Shock Breakout with Serendipitous GALEX Detections of Two SNLS Type II-P Supernovae, ApJ 683 (2008) L131 [arXiv:0804.1123].

[16] K. Schawinski, S. Justham, C. Wolf, P. Podsiadlowski, M. Sullivan, K. C. Steenbrugge, T. Bell, H. -J. Roeser et al., Supernova shock breakout from a red supergiant, Science 321 (2008) 223 [arXiv:0803.3596].

[17] M. Modjaz, W. Li, N. Butler, R. Chornock, D. Perley, S. Blondin, J. S. Bloom, A. V. Filippenko et al., From shock breakout to peak and beyond: Extensive panchromatic observations of the aspherical type Ib supernova $2008 D$ associated with Swift X-ray transient 080109, ApJ 702 (2009) 226 [arXiv:0805.2201].

[18] P. A. Mazzali, S. Valenti, M. Della Valle, G. Chincarini, D. N. Sauer, S. Benetti, E. Pian, T. Piran et al., The metamorphosis of Supernova SN2008D/XRF080109: a link between Supernovae and GRBs/Hypernovae, Science 321 (2008) 1185 [arXiv:0807.1695].

[19] A. M. Soderberg, E. Berger, K. Page, P. Schady, J. Parrent, D. Pooley, X. -Y. Wang, E. Ofek et al., An extremely luminous X-ray outburst marking the birth of a normal supernova, Nature 453 (2008) 469 [arXiv:0802.1712].

[20] E. O. Ofek, I. Rabinak, J. D. Neill, I. Arcavi, S. B. Cenko, E. Waxman, S. R. Kulkarni, A. G. Yam et al., Supernova PTF 09uj: A possible shock breakout from a dense circumstellar wind, ApJ 724 (2010) 1396 [arXiv:1009.5378]. 
[21] S. Gezari, D. O. Jones, N. E. Sanders, A. M. Soderberg, T. Hung, S. Heinis, S. J. Smartt, A. Rest et al., GALEX Detection of Shock Breakout in Type II-P Supernova PS1-13arp: Implications for the Progenitor Star Wind, ApJ 804 (2015) 28 [arXiv:1502.06964].

[22] E. Waxman and A. Loeb, TeV neutrinos and GeV photons from shock breakout in supernovae, Phys. Rev. Lett. 87 (2001) 071101 [astro-ph/0102317].

[23] R. A. Chevalier and C. Fransson, Shock breakout emission from a type Ib/c supernova: XRF 080109/SN 2008D, ApJ 683 (2008) L135 [arXiv:0806.0371].

[24] E. O. Ofek, M. Sullivan, S. B. Cenko, M. M. Kasliwal, A. Gal-Yam, S. R. Kulkarni, I. Arcavi, L. Bildsten et al., An outburst from a massive star 40 days before a supernova explosion, Nature 494 (2013) 65 [arXiv:1302.2633].

[25] P. A. Crowther, Physical properties of Wolf-Rayet stars, ARA\&A 45 (2007) 177 [astro-ph/0610356].

[26] N. Langer, Pre-supernova evolution of massive single and binary stars, ARA\&A $\mathbf{5 0}$ (2012) 107 [arXiv:1206.5443].

[27] B. Katz, N. Sapir and E. Waxman, X-rays, gamma-rays and neutrinos from collisionless shocks in supernova wind breakouts, Proc. of the International Astronomical Union 7 (2011) 274 [arXiv:1106.1898].

[28] R. A. Chevalier and C. M. Irwin, Shock breakout in dense mass loss: Luminous supernovae, ApJ 729 (2011) L6 [arXiv:1101.1111].

[29] R. A. Chevalier and C. M. Irwin, X-rays from supernova shocks in dense mass loss, ApJ 747 (2012) L17 [arXiv:1201.5581].

[30] K. Murase, T. A. Thompson, B. C. Lacki and J. F. Beacom, New class of high-energy transients from crashes of supernova ejecta with massive circumstellar material shells, Phys. Rev. D 84 (2011) 043003 [arXiv:1012.2834].

[31] G. Svirski, E. Nakar and R. 'e. Sari, Optical to X-rays supernovae light curves following shock breakout through a thick wind, ApJ 759 (2012) 108 [arXiv:1202.3437].

[32] K. Kashiyama, K. Murase, S. Horiuchi, S. Gao and P. Meszaros, High energy neutrino and gamma ray transients from relativistic supernova shock breakouts, ApJ 769 (2013) L6 [arXiv:1210.8147].

[33] A. R. Bell, K. Schure, B. Reville and G. Giacinti, Cosmic ray acceleration and escape from supernova remnants, MNRAS 431 (2013) 415 [arXiv:1301.7264].

[34] G. Svirski and E. Nakar, SN 2008D: A Wolf-Rayet explosion through a thick wind, ApJ 788 (2014) L14 [arXiv:1403.3400]. 